\title{
The progression of epithelial-mesenchymal transformation in gliomas
}

\author{
Lei Du, Jun-Hai Tang, Guo-Hao Huang, Yan Xiang and Sheng-Qing Lv*
}

\begin{abstract}
Epithelial-mesenchymal transformation(EMT) is a coordinated process in which polarized epithelial cells are induced to lose adhesion from the basement membrane and obtain the properties of mesenchymal cells, including invasion and metastasis. It has been proved that EMT greatly contributes to the invasion and therapeutic resistance of various solid human cancers. However, the role of EMT in brain glioma has not yet been fully clarified. So in this review, we mainly elaborate the latest progression about the related regulatory transcription factors, key signaling pathways and microRNAs (miRNAs) of EMT in gliomas.
\end{abstract}

Keywords: Brain glioma, Epithelial-mesenchymal transformation(EMT), Transcription factors, Signaling pathways, MicroRNAs

\section{Background}

Epithelial-mesenchymal transformation(EMT) was first observed in breast cancer in 1890s. Zhang J. et al. found that the EMT and mesenchymal-to-epithelial transition (MET, its reverse process), were reduplicative and alternatively occurred in chicken's epithelial cells [1]. In the recent 30 years, EMT becomes a hot research field and is generally divided into three different subtypes [2]. While type 1 EMT takes effect in the process of embryonic development and differentiation. Type 2 EMT mainly appears in the process of adult inflammation and tissue repair in the condition of wound healing and organ fibrosis. Type 3 EMT is active in tumorigenesis, involving the acquisition of new phenotype, enhancement of motility and lose of the original morphology, and also a diversified dynamic process regulated by multiple factors [3].

Almost all kinds of cancers will undergo invasion and metastasis with transformation from epithelial phenotype (marked with E-cadherin, Claudin) to mesenchymal phenotype (marked with N-cadherin, Vimentin, Fibronectin, etc), which is similar to the features of EMT. Therefore, EMT is considered as a potential target in cancer diagnosis and treatment, and the critical function of EMT in tumor formation and metastasis has been intensely studied.

\footnotetext{
* Correspondence: Ivsq0518@hotmail.com

Department of Neurosurgery, Xinqiao Hospital, Third Military Medical University, Chongqing 400037, People's Republic of China
}

Gliomas are the most common primary malignant brain cancers with an extremely high mortality. Despite the great efforts in both clinical and basic research, the prognosis of malignant gliomas remains quite poor. As the most aggressive and lethal malignant gliomas (WHO grade IV), glioblastoma multiforme (GBM) are intractable and resistant to the comprehensive therapy that consists of surgery, radiotherapy and chemotherapy. So the imperative task is to antagonize gliomas in a better way. Targeting EMT has demonstrated an anti-neoplasm effect in many solid cancers like hepatocellular and breast cancers. However, the role of EMT in gliomas has been rarely studied so far. In this review, we mainly discussed some key factors relating to EMT in gliomas, each seemingly working through its individual pathway, such as essential transcriptinal factors (Snail, Slug, Twist, etc), signaling pathways (Notch and Wnt signal pathway, etc), miRNAs and tumor hypoxia microenvironment. Long-noncoding RNA as a new field of study, appears in a small number of references in the article. And the critical connection or crosstalk between these factors were also discussed (Fig. 1).

\section{EMT-transcriptional factors(EMT-TFs) in Glioma}

EMT is a dynamic process regulated by many kinds of specific transcription factors, called EMT-TFs, such as Twist, nuclear factor-kappa $\mathrm{B}(\mathrm{NF}-\mathrm{kB})$ and the Zinc Finger E-box-binding Homeobox(ZEB1), which can down-regulate the metastasis-suppressor E-cadherin and up-regulate mesenchymal phenotype. 


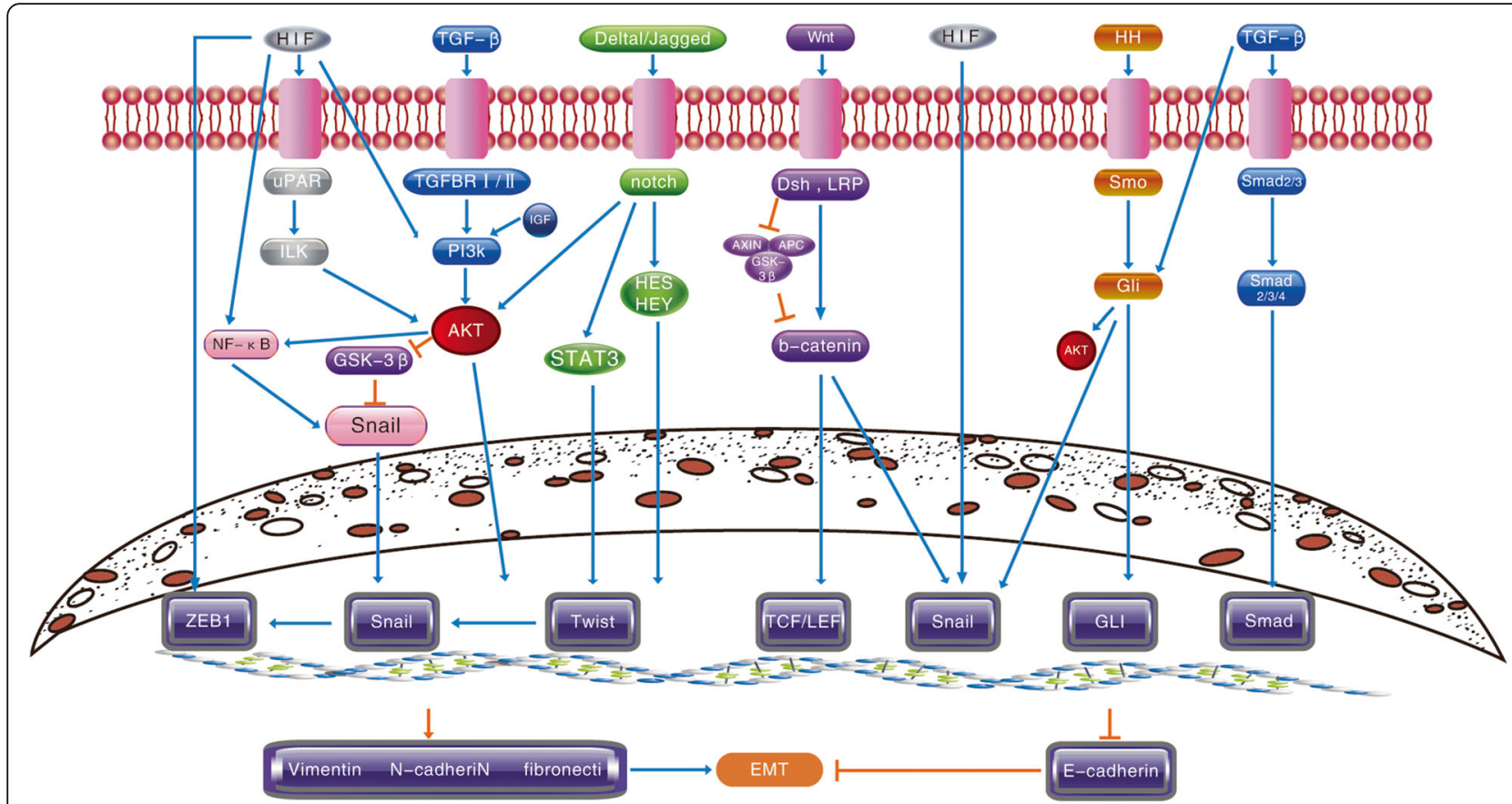

Fig. 1 The relative regulatory transcription factors, key signaling pathways and microRNAs(miRNAs) of EMT in gliomas

\section{Snail}

Snail was firstly found participate in the mesoderm formation of Drosophila melanogaster. Three Snail family proteins have been identified in vertebrates: Snail, Slug and Smuc. Snail and Slug, as two master regulators of the epithelial-mesenchymal transition, mainly mediate E-cadherin repression and are overexpressed in glioma cells during EMT. Snail also can be induced by TGF- $\beta$, Notch and has crosstalk with many signaling molecules. Snail correlates with the tumor grade and invasion, and the high expression of Snail indicates a poor prognosis in glioma [4]. Researchers find that the Sonic Hedgehog/ GLI signaling pathway directly participates in upregulation of Snail [5]. Evidence sustains that Snail is also the target of EMT-related protein, like IL-8 can induced glioma cells invasion by increasing NF-kB and Snail [6]. Intriguingly, autophagy can achieve GBM cells from mesenchymal phenotype to epithelial-like phenotype by down-regulation of snail and slug [7].

\section{ZEB1}

ZEB proteins contain ZEB1 and ZEB2. ZEB1 is responsible for DNA binding and ZEB2 is known as SMAD1interacting protein, high levels of which involves in malignant transformation. As a dominating metastasis factor, ZEB1 suppresses E-cadherin, resulting in the loss of cell-cell adhesion and gain of mesenchymal characteristics, ZEB1 is associated with glioma invasion and poor prognosis. What's more, further study also reveals that in IDH1-mutant lower-grade gliomas ZEB1 expression is relatively increased [8]. In gliomas,ZEB1 renders EMT via activating PDGFA/PDGFR $\alpha$ signaling, leading to the enhancement of tumor growth and invasion [9]. ZEB1 also contributes to therapeutic resistance in various cancers via EMT-dependent or EMT-independent mechanisms. One study confirms CBX7 can block ZEB1 expression and suppress EMT in LN229, T98G cells and primary glioma cells [10].

\section{NF-KB}

The mammalian transcription factor NF- $\mathrm{BB}$ family also involves in the process of EMT. In canonical pathway, $N F-\kappa B$ inhibitory protein $(\mathrm{I} \kappa \mathrm{B})$ is phosphorylated by the $\mathrm{I}_{\kappa} \mathrm{B}$ kinase complex, resulting in the activation of NF- $\kappa \mathrm{B}$. Activated NF- $\mathrm{kB}$ translocates to nuclear and initiates transcprition of different target genes. A series of experiments show that NF- $\mathrm{kB}$ induces EMT and metastasis, inhibits apoptosis in numerous kinds of tumor cells. Accumulating evidences show that PI3K/Akt or MAPK ERK pathways can trigger the NF- $\mathrm{KB}$ signaling. SENP1, a member of SENPs family, which play a pro-oncogenic role in many types of cancer including glioma, can work as a upstream of NF- $\kappa$ B pathway [11]. In addition, IL-8, a new prognostic factor for glioma, can activate EMT through ELMO1-NF-kB-Snail signaling [6]. As a upstream factor, NF- $\mathrm{kB}$ can also enhance other key factors, such as Snail and Yin Yang 1 (YY1). The interaction described above forms a Snail/NF-кB/YY1/RKIP circuit, which promotes metastasis and restrains apoptosis. The loop can be inhibited by the Nitric Oxide via 
nitrosylation of NF-kB, YY1 and Snail, consequently leading to EMT suppression [12]. In tumor treatment, Piperlongumine has been a new pharmacotherapy for glioma, which can selectively kill GBM cells but not normal astrocytes [13].

\section{Twist}

Twist consists of two proteins: Twist1 and Twist2, which have a basic helix-loop-helix (bHLH)structure. Although the upstream or downstream signaling pathways of Twist are not completely elucidated yet, Twist has been found important in cancer metastasis, tissue fibrosis, and early embryonic morphogenesis. Usually Twist is identified as Twist1 and Twist1 is thought more significant in cancer metastasis than Twist2. Twist1 is upregulated in multiple carcinomas and indicates a poor prognosis [14]. In the progression of cancer, Twist acts independently of Snail to suppress E-cadherin and upregulate $\mathrm{N}$-cadherin and fibronectin. At present the well-known upstream and downstream factors of Twist pathway are abundant and on the rise. It has been verified that Notch has a significant association with Twist through a Notch1/STAT3/Twist signaling axis. Moreover, Akt is found to be another vital upstream factor of Twist [15]. Twist is also a target for new treatment due to its important function in EMT. For instance, cell differentiation agent-2 (CDA-2) was used to suppressing Twist/Slug signaling in glioma [16]. IQGAP1 is aberrantly expressed in several tumor types. Recently IQGAP1-small interfering (si)RNA is discovered to inhibit U251 and U373 cells through inhibition of Snail and Twist, but the impact on EMT is not elaborated [17].

\section{Signaling pathways involving in Glioma EMT}

A number of different molecular processes are jointly activated to initiate EMT and make it develop orderly, consequently achieving the transformation from the polarized epithelial cells to mesenchymal cells.

\section{Notch signaling pathway}

Notch signaling pathway, which acts on embryonic development, is a key pathway in EMT. In mammals, five canonical transmembrane ligands (Delta1/3/4, Jagged1/ 2) and four transmembrane Notch receptors (Notch $1 / 2 /$ 3/4) are expressed. After complex biochemical process, the Notch pathway finally activate HES and HEY family. Studies have demonstrated that Notch1 promotes tumor progression via regulating EMT correlative factors (activating E-cadherin, inhibiting N-cadherin, Vimentin) [18]. Recent findings elucidated that inhibitor of Notch1 restrained invasion and migration of glioma cells via inhibiting Notch1-HES1 signaling, and simultaneously induced cell autophagy [19]. Previous studies have illustrated that Notch1 and Jagged1 could depress the Ecadherin by regulating Slug and Snail, then facilitating the EMT. Moreover, Notch1 inhibitor suppresses the expression of Slug and Snail. TUG1, as a downstream target of Notch, can maintains tumorigenicity and stemness features of glioma stem cells (GSCs) [20]. Acting as a promoter of epithelial-mesenchymal tranformation in glioma, ZFAS1 also activites Notch signaling pathways [21]. Recent researchers focus on non-coding RNA which correlates with notch, one study have uncovered that miR-107 restrained invasion and migration via regulating Notch2 expression in glioma cells [22], but the whole mutual effect between Notch and other factors needs further investigation.

\section{WNT signaling pathway}

In many types of cancer, abnormal activation or mutations in the WNT pathway have been reported. Emerging evidence has suggested that deregulation of the Wnt pathway is relevant to brain tumors. In canonical Wnt signaling pathway, combination of Wnts with receptor Frizzled is the mark of cascade stimulation, in which LRP provides assistance. Frizzled recruits Disheveled (Dsh) and LRP recruits Axin, contributing to the formation of downstream degradation complex. The degradation complex comprises of Axin, glycogen synthase kinase (GSK-3 $\beta$ ) and APC, which together induce $\beta$-catenin phosphorylation and inhibit the degradation of $\beta$-catenin. Then accumulating $\beta$-catenin translocates to the nucleus where it activates the transcriptional factors TCF/LEF then regulate target genes referring to cellular proliferation, differentiation, survival, and apoptosis [23]. Previous data reported that Wnt2 and Wnt5a was expressed in gliomas, and knockout of Wnt2 in human U251 glioma cells inhibited cell proliferation, invasive ability, and induces apoptosis [24]. Another experiment showed that the expression of Wnt5a was positively correlated with the proliferation of glioma cells in vitro. Moreover,Frizzled 4 promotes EMT by regulating Snail1, resulting in acquisitions of mesenchymal phenotype, neurosphere formation and invasion [25]. Furthermore, there is also a positive feedback between Snail 1 and $\beta$-catenin. Another study showed that HOXA13 may be a potential diagnostic biomarker for glioma which induce progression of glioma in TGF- $\beta$ or Wnt-dependent way [26]. And Wnt/ß-catenin signaling also can be depressed by IDH1-R132H in vitro. As a member of long noncoding RNA(lncRNA) family, CCND2-AS1 Synchronizes with Wnt/b-catenin signaling and the highly expression of CCND2-AS1 activates cell proliferation in glioma [27].In the opposite, another lncRNA, thyroid carcinoma susceptibility candidate 3 (PTCSC3) keeps a low level in glioma cells, and it can suppress the $\mathrm{Wnt} / \beta$-catenin signaling pathway and then inhibit EMT of glioma [28]. 


\section{Hh signaling pathway}

Hedgehog family members are of great importance in regulating the embryo-genesis, EMT and tumorigenesis. Hedgehog signaling contains three primary signaling proteins: Desert Hedgehog (Dhh), Indian Hedgehog (Ihh), and Sonic Hedgehog(Shh). In inactive state, downstream 7-pass transmembrane protein Smoothened(Smo) bonds to the Patched family members(including PTCH1 and PTCH2)) which are the receptor of Hedgehog. After Hedgehog binding to Patched family receptors, Smoothened is released from the Patched-dependent suppression, and the target gene GLI is activited through the SmoothenedGLI signaling cascade [29]. Previous studies have found that the Hedgehog signals indirectly induced EMT via relevant target gene JAG2 and up-regulating EMT regulators [30]. Twist1, similar to Snail is another direct target gene of Gli. Hh signaling pathway has complex crosstalk with other factors. A recent study revealed that TGF- $\beta 1$ is a key upstream factor of Shh signaling [31]. Meanwhile, the negative regulation of Wnt pathway by Gli activity has been described in human cancers cells. Gli1 and Gli3R were shown to suppress Wnt signaling pathway and then inhibit the proliferation of cancer cells. Together these facts indicate that Hh pathway is important in EMT and deserves further study [32].

\section{TGF- $\beta$ Signaling pathway}

The secretive protein TGF- $\beta$ is a wide key factor in EMT. TGF- $\beta$ is well conserved in evolution, and it regulates multiple cell phenotypes such as adhesion, growth, apoptosis, differentiation and fibrosis [33]. In human cells, the TGF- $\beta$ signaling pathway contains three ligand isoforms TGF- $\beta 1 / 2 / 3$ ) and two TGF- $\beta$ transmembrane receptors (TGFBR I/II). TGF- $\beta$ invokes many downstream pathways. But the only specific downstream target for the TGF- $\beta$ family is SMAD. Two molecules of phosphorylated SMADs(SMAD2/3) and a commonmediator SMAD(SMAD4) forms one trimer. Then the trimer will activate target genes through binding to specific EMT-TFs, individually or with other co-activators, and finally perform on biological process [34]. There is also the inhibitory SMADs (SMAD6/7) which will compose a negative segment in the cascade of TGF- $\beta$ pathway by competing with SMAD4 [35]. There are also several other TGF- $\beta$ pathways, such as TGF- $\beta$ /TRAF6/ p38/Jun or TGF- $\beta /$ RhoA axis. What deserves to be mentioned is that no matter in canonical or noncanonical TGF- $\beta$ pathway, TGF- $\beta$ participates in driving EMT. Receptor-associated SMADs interact with EMT-TFs such as AP-1 and Snail [36].TGF- $\beta 1$ treatment induces a significant change of cellular morphology depicted by increasing $\alpha$-SMA and $\mathrm{N}$-cadherin and corresponding decreased E-cadherin via a SMAD2/SMAD3-dependent manner. Notably, $\alpha$-SMA and N-cadherin increases are dependent on both SMAD2 and SMAD3, whereas Ecadherin is down-regulated by TGF- $\beta 1$ only in a SMAD3-dependent manner [37]. Some studies have confirmed that E-cadherin also could be suppressed by TGF- $\beta$ through ZEB, Snail and HMGA2. Another interesting finding is the discrepant expression of TGF- $\beta$ existing between the necrotic and non-necrotic areas of many tumors [33].

\section{PI3K/Akt signaling pathway}

Akt is a key intermediate activator and involved in several cascades regulating EMT-TFs in gliomas. In consideration of the complex regulation mechanism, we will clarify this cascade based on PI3K/Akt signaling pathway, which has been widely investigated for its effects in EMT and tumorigenesis. Akt can be activated by diversified stimuli such as phosphoinositide 3-kinase (PI3K), integrin-linked kinase (ILK), extracellular matrix components (ECM), growth factors (TGF- $\beta$ ate, HDGF) via phosphorylation events in cytoplasm. As a core activator, PI3K generates phosphatidylinositol-3,4,5-trisphosphate (PIP3) and leads to phosphorylation in Thr308 and partial activation of Akt. Then the full activation of Akt is marked with phosphorylation of Akt-Ser473. Activated Akt will start physiological processes, including apoptosis, metabolism and migration [38]. Recent research reports that high level of p-AKT is associated with worse overall survival in gliomas [39]. For example, GSK-3 $\beta$ as a constituent part of degradation complex in Wnt/ $\beta$-catenin signaling can be degraded after PI3K/Akt activation. On the other hand, GSK-3 $\beta$ participates in the degradation of Snail, which means that PI3K/Akt pathway participates in Wnt/ß-catenin signaling and Snail activation. An recent study shows that radicol can suppresses Akt/mTOR/p70S6K pathway through GSK-3 $\beta$ activation and then induces apoptosis in temozolomideresistant glioma cell, which is undoubtedly an exciting news [40]. Another research reports that AKT takes effect in Notch1-induced activation of $\beta$-catenin and NF- $\mathrm{kB}$ in EMT of glioma cells,and inhibition of Akt will depress the process [41]. Furthermore, SHH-Gli1 signaling can activate Akt in the process of EMT in gliomas. IGF acts as a activator in PI3K/Akt signaling. Inhibition of Imp2 and Osthole were also found to block IGF1 and IGF2 respectively, resulting in restrain of GBM malignancy and EMT $[42,43]$. The increase of E-cadherin expression induced by matrin can be attributed to the inhibition of AKT signaling [44]. Apigenin which has been mentioned before can inhibit phosphorylation of AKT Ser473 and attenuate atherogenesis through inducing macrophage apoptosis [45]. 


\section{EMT-related MicroRNAs in Gliomas}

MicroRNAs (miRNAs) are small noncoding RNAs which play fundamental roles in modulating the expression of target RNAs by initiating mRNA degradation or inhibiting protein translation. At present we know there exists a large amount of miRNAs relating to EMT in glioma. Microarray analysis proves that the miR-205 and miR200 family may be crucial in EMT. The overexpression of miR-200 clusters inhibits EMT via up-regulating Ecadherin and targeting the ZEB. What's more, ectopic expression of miR-200 in mesenchymal cells induced MET [46]. The later research showed the existence of an autocrine TGF- $\beta / \mathrm{ZEB} / \mathrm{miR}-200$ signaling regulatory network that controlled the transformation between mesenchymal and epithelial status. Furthermore, miR-200 can cooperate with miR-205 to induce a MET process by suppressing ZEB [47]. Many other miRNAs also affect some EMT-TFs. MiR-590-3p suppresses cells migration and invasion by regulating ZEB1 and ZEB2 in GBM. For gliomas, miR-340 can dramatically induce cell cycle arrest and apoptosis, promote autophagy, suppress cell motility and inhibit glioma cell proliferation via inhibiting different targets such as ROCK1 or p-AKT [48]. Over-expression of miR-9 plays an core role in gliomas progression and predicts an unfavorable prognosis. Upregulation of miR-9 leads to suppression of Cadherin-1 (CDH1) and enhancement of cell motility and invasiveness [49]. Likewise, studies demonstrated that miR-204 could directly target TGF- $\beta$ RII and Snail2. A reduction in miR-204 expression suppressed Snail, which was rapidly induced by TGF- $\beta$ signaling during EMT [50]. In addition, a recent research found elevated miR-181, which consists of $181 \mathrm{a} / \mathrm{b} / \mathrm{c}$, indicates a better prognosis, could inhibit glioma cell invasion and proliferation via suppressing KPNA4 or TGF- $\beta$ and reverse EMT [51]. Two newly found MicroRNAs, mir-361-5p and mir-194 both significantly reduced in glioma cell lines, and overexpression of mir-361-5p and mir-194 inhibited EMT in glioma cell, respectively targeting their downstream Twist1 and Bmi1 $[52,53]$. Certain integrated studies list some microRNAs as follows: miR-10a,-124,-107,-222 and -221 can suppress mesenchymal markers and inhibit invasion. In contrast, miR-182, $-21,-130 \mathrm{~b}$ and -30e play an opposite role (Fig. 2), while the function of miR16 in the glioma progression is controversial.

\section{EMT microenvironment in Glioma}

There is no doubt that the microenvironment is responsible for tumor recurrence, metastasis and chemotherapy resistance. Hypoxia is a key microenvironment element in many solid tumors and promotes the development of EMT mainly by activating the related cytokines. Hypoxia inducible factors(HIFs) are the core effectors of hypoxia, which consist of HIF- $\alpha$ and HIF- $\beta$ [54]. HIF- $1 \alpha$ is most important in correlation with metastasis, prognostic outcomes in cancers. Knockdown of HIF-1 $\alpha$ remarkably enhances drug sensitivity and reduces tumor invasion and metastasis in glioma cells [55]. Studies have established the decisive role of HIF in EMT under the condition of hypoxia through the direct regulation of ZEB1 in glioma [56]. Some other hypoxia-induced factors like PLOD2 also can promote glioma cells proliferation, migration and invasion via change the expression of $\beta$-catenin, snail and slug [57]. A recent research discovered the inhibition of p75 neurotrophin receptor enhances EMT in glioma cells and there exists an inverse expression between p75NTR and HIF-1 $\alpha$ : HIF-1a over-expression could down-regulate the expression of p75NTR, which indicated that p75NTR might be a significant downstream factor of hypoxia-induced EMT [58]. The culminating expression of Urokinase receptor(UPAR) under hypoxia induces EMT, and intermittent hypoxia transcriptional inactivation of UPAR enhances the apoptotic response in medulloblastoma cells. A recent study reported that the mesenchymal EMT-TFs C/EBP-ßand C/EBP- $\delta$ were highly expressed in GBM, especially in necrotic zones suffering from hypoxic conditions [33]. Hypoxia associates with most processes of EMT and more mechanisms are being discovered, so targeting HIF can be a new method to restrain EMT. A recent research has tested

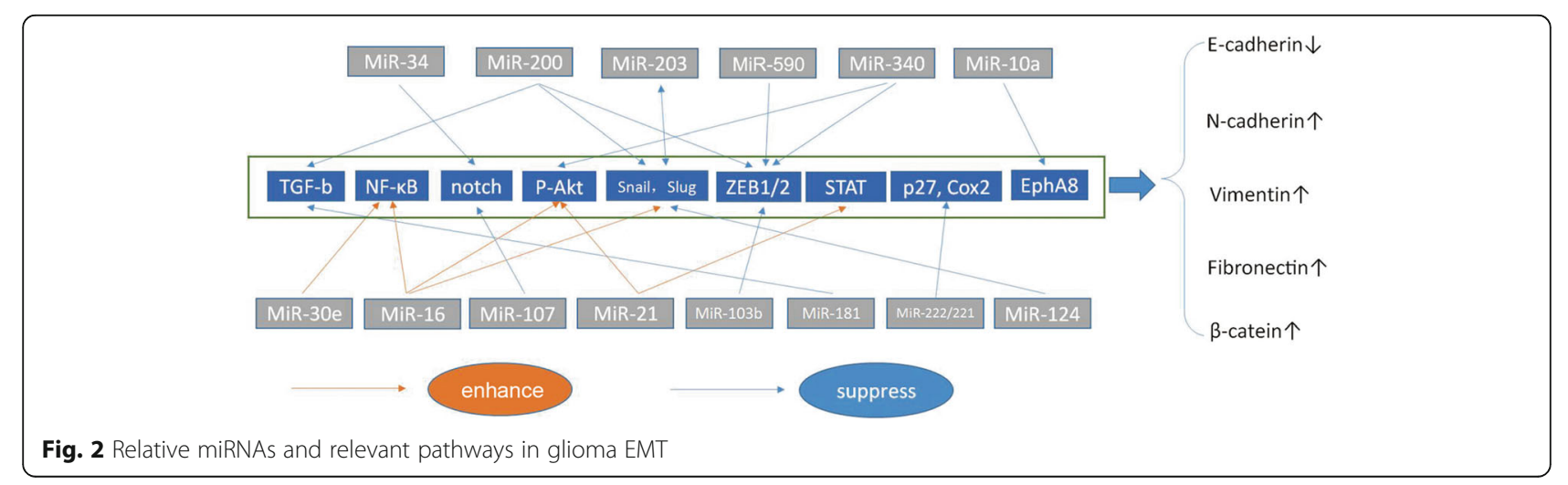


the HIF inhibitor as adjuvant therapy for anti-EMT therapy. Another study reports that hypoxia can activate EGFR/PI3K/AKT pathway and promote EMT, meanwhile LRIG1 can block the whole process [59].

\section{Conclusions}

In this review, we have made an introduction of some key factors involving in the EMT process. It has been well recognized that EMT are associated with aggressive tumor growth, distant colonization, and therapeutic resistance. EMT in malignant gliomas has attracted more and more attention. With the continuous efforts of many researchers in this field, great progresses have been made. Elucidation of the complex mechanisms underlying EMT in gliomas will improve the clinical treatment of this refractory cancer. At last, this review will offer some help for people to understand the EMT in brain gliomas.

\section{Abbreviations \\ bHLH: Basic helix-loop-helix; CDA-2: Cell differentiation agent-2; CDH1: Cadherin-1; Dhh: Desert Hedgehog; Dsh: Disheveled; ECM: Extracellular matrix components; EMT: Epithelial-mesenchymal transformation; GBM: Glioblastoma multiforme; GSCs: Glioma stem cells; GSK-3ß: Glycogen synthase kinase; Ihh: Indian Hedgehog; IKKa: The I-KB kinasea; ILK: Integrin-linked kinase; IncRNA: Long noncoding RNA; MET: mesenchymal-to-epithelial transition; miRNAs: MicroRNAs; NF-KB: Nuclear factor-kappa B; PI3K: Phosphoinositide 3-kinase; PIP3: Phosphatidylinositol-3,4,5- trisphosphate; Shh: Sonic Hedgehog; Smo: Smoothened; TGFBR: TGF- $\beta$ transmembrane receptors; UPAR: Urokinase receptor; YY1: Yin Yang 1; ZEB: The Zinc Finger E-box-binding Homeobox}

\section{Acknowledgements}

We thank Dr. Changjiang Hu from the Department of Gastroenterology, Xinqiao Hospital, Chongqing for his assistance in revising this manuscript.

\section{Funding}

The cost of this review, including manuscript writing and figures processing, was supported by the National Natural Science Foundation of China(NSFC-81272783).

\section{Availability of data and materials}

All data generated or analyzed during this study are included in this published article.

\section{Authors' contributions}

LD was responsible for collecting, sorting out the article and writing this review. JH T, GH H, YX, SQ L were kindly help draw the figure and revise review later. All authors read and approved the final manuscript.

\section{Authors' information}

First author: Lei Du, master student,

Second author: Jun-Hai Tang, doctor candidate

Third author: Guo-Hao Huang, doctor candidate

Fourth author: Yan Xiang, master student

Corresponding author: Professor Sheng-Qing LV*

Department of Neurosurgery, Xinqiao Hospital, Third Military Medical

University, Chongqing, 400,037, P.R. China

\section{Ethics approval and consent to participate}

Not applicable.

\section{Consent for publication}

Not applicable.

\section{Competing interests}

The authors declare that they have no competing interests.
Received: 16 December 2016 Accepted: 19 June 2017

Published online: 03 August 2017

\section{References}

1. Zhang J, Tian XJ, Xing J. Signal Transduction Pathways of EMT Induced by TGF-beta, SHH, and WNT and Their Crosstalks. J Clin Med. 2016;5(4):41.

2. Zaravinos A. The regulatory role of MicroRNAs in EMT and cancer. J Oncol. 2015;2015:865816.

3. Nieto MA, Huang RY, Jackson RA, Thiery JP. EMT: 2016. Cell. 2016;166(1):21.

4. Myung J, Cho BK, Kim YS, Park SH. Snail and Cox-2 expressions are associated with WHO tumor grade and survival rate of patients with gliomas. Neuropathology. 2010;30(3):224.

5. Wang K, Pan L, Che X, Cui D, Li C. Sonic hedgehog/GLI(1) signaling pathway inhibition restricts cell migration and invasion in human gliomas. Neurol Res. 2010;32(9):975.

6. Zhang B, Shi L, Lu S, Sun X, Liu Y, et al. Autocrine IL-8 promotes F-actin polymerization and mediate mesenchymal transition via ELMO1-NF-kappaBsnail signaling in glioma. Cancer Biol Ther. 2015;16(6):898.

7. Catalano M, D'Alessandro G, Lepore F, Corazzari M, Caldarola S, et al. Autophagy induction impairs migration and invasion by reversing EMT in glioblastoma cells. Mol Oncol. 2015;9(8):1612.

8. Nesvick CL, Zhang C, Edwards NA, Montgomery BK, Lee M, et al. ZEB1 expression is increased in IDH1-mutant lower-grade gliomas. J Neuro-Oncol. 2016;130(1):111.

9. Zhang L, Zhang W, Li Y, Alvarez A, Li Z, et al. SHP-2-upregulated ZEB1 is important for PDGFRalpha-driven glioma epithelial-mesenchymal transition and invasion in mice and humans. Oncogene. 2016;35(43):5641.

10. Bao Z, Xu X, Liu Y, Chao H, Lin C, et al. CBX7 negatively regulates migration and invasion in glioma via Wnt/beta-catenin pathway inactivation. Oncotarget. 2017;8(24):39048.

11. Xia W, Tian H, Cai X, Kong H, Fu W, et al. Inhibition of SUMO-specific protease 1 induces apoptosis of astroglioma cells by regulating NF-kappaB/ Akt pathways. Gene. 2016;595(2):175.

12. Bonavida B, Baritaki S. Inhibition of epithelial-to-Mesenchymal transition (EMT) in cancer by nitric oxide: pivotal roles of Nitrosylation of NF-kappaB, YY1 and snail. Forum Immunopathol Dis Ther. 2012;3(2):125.

13. Liu QR, Liu JM, Chen Y, Xie XQ, Xiong XX, et al. Piperlongumine inhibits migration of glioblastoma cells via activation of ROS-dependent p38 and JNK signaling pathways. Oxidative Med Cell Longev. 2014;2014:653732.

14. Wushou A, Hou J, Zhao YJ, Shao ZM. Twist-1 up-regulation in carcinoma correlates to poor survival. Int J Mol Sci. 2014;15(12):21621.

15. Vichalkovski A, Gresko E, Hess D, Restuccia DF, Hemmings BA. PKB/AKT phosphorylation of the transcription factor Twist-1 at Ser42 inhibits p53 activity in response to DNA damage. Oncogene. 2010;29(24):3554.

16. Xie YK, Huo SF, Zhang G, Zhang F, Lian ZP, et al. CDA-2 induces cell differentiation through suppressing Twist/SLUG signaling via miR-124 in glioma. J Neuro-Oncol. 2012;110(2):179.

17. Diao B, Liu Y, Zhang Y, Yu J, Xie J, et al. IQGAP1siRNA inhibits proliferation and metastasis of U251 and U373 glioma cell lines. Mol Med Rep. 2017;15(4):2074.

18. Espinoza I, Miele L. Deadly crosstalk: notch signaling at the intersection of EMT and cancer stem cells. Cancer Lett. 2013;341(1):41.

19. Yao J, Zheng K, Li C, Liu H, Shan X. Interference of Notch1 inhibits the growth of glioma cancer cells by inducing cell autophagy and downregulation of Notch1-Hes-1 signaling pathway. Med Oncol (Northwood, London, England). 2015;32(6):610.

20. Katsushima K, Natsume A, Ohka F, Shinjo K, Hatanaka A, et al. Targeting the notch-regulated non-coding RNA TUG1 for glioma treatment. Nat Commun. 2016;7:13616.

21. Gao K, Ji Z, She K, Yang Q, Shao L. Long non-coding RNA ZFAS1 is an unfavourable prognostic factor and promotes glioma cell progression by activation of the notch signaling pathway. Biomed Pharmacother. 2017;87:555.

22. Chen L, Chen XR, Zhang R, Li P, Liu Y, et al. MicroRNA-107 inhibits glioma cell migration and invasion by modulating Notch2 expression. J NeuroOncol. 2013;112(1):59.

23. Pu P, Zhang Z, Kang C, Jiang R, Jia Z, et al. Downregulation of Wnt2 and beta-catenin by siRNA suppresses malignant glioma cell growth. Cancer Gene Ther. 2009;16(4):351.

24. Kim KH, Seol HJ, Kim EH, Rheey J, Jin HJ, et al. Wnt/beta-catenin signaling is a key downstream mediator of MET signaling in glioblastoma stem cells. Neuro-Oncology. 2013;15(2):161. 
25. Jin $\mathrm{X}$, Jeon HY, Joo KM, Kim JK, Jin J, et al. Frizzled 4 regulates stemness and invasiveness of migrating glioma cells established by serial intracranial transplantation. Cancer Res. 2011;71(8):3066.

26. Duan R, Han L, Wang Q, Wei J, Chen L, et al. HOXA13 is a potential GBM diagnostic marker and promotes glioma invasion by activating the Wnt and TGF-beta pathways. Oncotarget. 2015;6(29):27778.

27. Zhang H, Wei DL, Wan L, Yan SF, Sun YH. Highly expressed IncRNA CCND2AS1 promotes glioma cell proliferation through Wnt/beta-catenin signaling. Biochem Biophys Res Commun. 2017;482(4):1219.

28. Xia S, Ji R, Zhan W. Long noncoding RNA papillary thyroid carcinoma susceptibility candidate 3 (PTCSC3) inhibits proliferation and invasion of glioma cells by suppressing the Wnt/beta-catenin signaling pathway. BMC Neurol. 2017;17(1):30.

29. Ruiz i Altaba A, Mas C, Stecca B. The Gli code: an information nexus regulating cell fate, stemness and cancer. Trends Cell Biol. 2007;17(9):438.

30. Katoh Y, Katoh M. Hedgehog signaling, epithelial-to-mesenchymal transition and miRNA (review). Int J Mol Med. 2008;22(3):271.

31. Islam SS, Mokhtari RB, Noman AS, Uddin M, Rahman MZ, et al. Sonic hedgehog (Shh) signaling promotes tumorigenicity and stemness via activation of epithelial-to-mesenchymal transition (EMT) in bladder cancer. Mol Carcinog. 2016;55(5):537.

32. Onishi $\mathrm{H}$, Katano M. Hedgehog signaling pathway as a therapeutic target in various types of cancer. Cancer Sci. 2011;102(10):1756.

33. Iwadate $Y$, Matsutani T, Hirono S, Shinozaki N, Saeki N. Transforming growth factor-beta and stem cell markers are highly expressed around necrotic areas in glioblastoma. J Neuro-Oncol. 2016;129(1):101.

34. Wu MY, Hill CS. Tgf-beta superfamily signaling in embryonic development and homeostasis. Dev Cell. 2009;16(3):329.

35. Massague J, Seoane J, Wotton D. Smad transcription factors. Genes Dev. 2005;19(23):2783.

36. Fuxe J, Vincent T, Garcia de Herreros A. Transcriptional crosstalk between TGF-beta and stem cell pathways in tumor cell invasion: role of EMT promoting Smad complexes. Cell cycle (Georgetown, Tex). 2010; 9(12):2363.

37. Islam SS, Mokhtari RB, El Hout Y, Azadi MA, Alauddin M, et al. TGF-beta1 induces EMT reprogramming of porcine bladder urothelial cells into collagen producing fibroblasts-like cells in a Smad2/Smad3-dependent manner. J Cell Commun Signaling. 2014;8(1):39.

38. Chautard E, Ouedraogo ZG, Biau J, Verrelle P. Role of Akt in human malignant glioma: from oncogenesis to tumor aggressiveness. J NeuroOncol. 2014;117(2):205.

39. Antonelli M, Massimino M, Morra I, Garre ML, Gardiman MP, et al. Expression of pERK and PAKT in pediatric high grade astrocytomas: correlation with YKL40 and prognostic significance. Neuropathology. 2012;32(2):133.

40. Li ZY, Zhang C, Chen L, Chen BD, Li QZ, et al. Radicol, a novel Trinorguaiane-type Sesquiterpene, induces Temozolomide-resistant Glioma cell apoptosis via ER stress and Akt/mTOR pathway blockade. Phytother Res. 2017;31(5):729.

41. Zhang X, Chen T, Zhang J, Mao Q, Li S, et al. Notch1 promotes glioma cell migration and invasion by stimulating beta-catenin and NF-kappaB signaling via AKT activation. Cancer Sci. 2012;103(2):181.

42. Lin YC, Lin JC, Hung CM, Chen Y, Liu LC, et al. Osthole inhibits insulin-like growth factor-1-induced epithelial to mesenchymal transition via the inhibition of PI3K/Akt signaling pathway in human brain cancer cells. J Agric Food Chem. 2014;62(22):5061.

43. Mu Q, Wang L, Yu F, Gao H, Lei T, et al. Imp2 regulates GBM progression by activating IGF2/PI3K/Akt pathway. Cancer Biol Ther. 2015;16(4):623.

44. Wang $Z$, Wu $Y$, Wang $Y$, Jin $Y$, Ma $X$, et al. Matrine inhibits the invasive properties of human glioma cells by regulating epithelialtomesenchymal transition. Mol Med Rep. 2015;11(5):3682.

45. Zeng P, Liu B, Wang Q, Fan Q, Diao JX, et al. Apigenin attenuates Atherogenesis through inducing macrophage apoptosis via inhibition of AKT Ser473 Phosphorylation and Downregulation of Plasminogen activator inhibitor-2. Oxidative Med Cell Longev. 2015;2015:379538.

46. Gregory PA, Bert AG, Paterson EL, Barry SC, Tsykin A, et al. The miR-200 family and miR-205 regulate epithelial to mesenchymal transition by targeting ZEB1 and SIP1. Nat Cell Biol. 2008;10(5):593.

47. Braun J, Hoang-Vu C, Dralle H, Huttelmaier S. Downregulation of microRNAs directs the EMT and invasive potential of anaplastic thyroid carcinomas. Oncogene. 2010;29(29):4237.

48. Huang D, Qiu S, Ge R, He L, Li M, et al. miR-340 suppresses glioblastoma multiforme. Oncotarget. 2015;6(11):9257.
49. Wu Z, Wang L, Li G, Liu H, Fan F, et al. Increased expression of microRNA-9 predicts an unfavorable prognosis in human glioma. Mol Cell Biochem. 2013;384(1-2):263

50. Lin X, Yang B, Liu W, Tan X, Wu F, et al. Interplay between PCBP2 and miRNA modulates ARHGDIA expression and function in glioma migration and invasion. Oncotarget. 2016;7(15):19483.

51. Wang $H$, Tao $T$, Yan W, Feng $Y$, Wang $Y$, et al. Upregulation of miR-181s reverses mesenchymal transition by targeting KPNA4 in glioblastoma. Sci Rep. 2015;5:13072.

52. Zhang X, Wei C, Li J, Liu J, Qu J. MicroRNA-194 represses glioma cell epithelialtomesenchymal transition by targeting Bmi1. Oncol Rep. 2017;37(3):1593.

53. Zhang X, Wei C, Li J, Liu J, Qu J. MicroRNA-361-5p inhibits epithelial-tomesenchymal transition of glioma cells through targeting Twist1. Oncol Rep. 2017;37(3):1849.

54. Semenza GL. HIF-1 mediates metabolic responses to intratumoral hypoxia and oncogenic mutations. J Clin Invest. 2013;123(9):3664.

55. Tang JH, Ma ZX, Huang GH, Xu QF, Xiang Y, et al. Downregulation of HIF-1a sensitizes U251 glioma cells to the temozolomide (TMZ) treatment. Exp Cell Res. 2016;343(2):148.

56. Joseph JV, Conroy S, Pavlov K, Sontakke P, Tomar T, et al. Hypoxia enhances migration and invasion in glioblastoma by promoting a mesenchymal shift mediated by the HIF1alpha-ZEB1 axis. Cancer Lett. 2015;359(1):107.

57. Song $Y$, Zheng S, Wang J, Long H, Fang L, et al. Hypoxia-induced PLOD2 promotes proliferation, migration and invasion via PI3K/Akt signaling in glioma. Oncotarget. 2017;

58. Wang TC, Luo SJ, Lin CL, Chang PJ, Chen MF. Modulation of p75 neurotrophin receptor under hypoxic conditions induces migration and invasion of C6 glioma cells. Clin Exp Metastasis. 2015;32(1):73.

59. Zhang X, Song Q, Wei C, Qu J. LRIG1 inhibits hypoxia-induced vasculogenic mimicry formation via suppression of the EGFR/PI3K/AKT pathway and epithelial-to-mesenchymal transition in human glioma SHG-44 cells. Cell Stress Chaperones. 2015;20(4):631.

\section{Submit your next manuscript to BioMed Central and we will help you at every step:}

- We accept pre-submission inquiries

- Our selector tool helps you to find the most relevant journal

- We provide round the clock customer support

- Convenient online submission

- Thorough peer review

- Inclusion in PubMed and all major indexing services

- Maximum visibility for your research

Submit your manuscript at www.biomedcentral.com/submit
) Biomed Central 